\title{
Plant species of Okhla Bird Sanctuary: A wetland of Upper Gangetic Plains, India
}

\author{
Upma Manral, Angshuman Raha, Ridhima Solanki, Syed Ainul Hussain*, Mattozbiyil Mani Babu, \\ Dhananjai Mohan, Gopi G. Veeraswami, K. Sivakumar and Gautam Talukdar \\ Wildlife Institute of India, Post Box \# 18, Chandrabani, Dehra Dun. 248001. Uttarakhand, India. \\ * Corresponding author. E-mail: hussain@wii.gov.in
}

\begin{abstract}
The Okhla Bird Sanctuary (OBS), a man-modified floodplain wetland having high human impact, is located in an urbanized landscape. Its location in the Central Asian Flyway of migratory birds makes it an ideal transit and wintering ground for birds. This paper describes the vegetation composition and significance of the Sanctuary as a bird habitat. A floristic survey was carried out from winter 2009 to spring 2010 while preparing a management plan for OBS. 192 species of plants belonging to 46 families were recorded from the area. Thirteen species of weeds were observed covering $70 \%$ of the sampled area in peak summer. Among trees, highest density was found for two exotic species viz., Leucaena leucocephala and Prosopis juliflora. The aquatic system is facing threat from proliferation of Eichhornia cressipes and Typha angustifolia. Strategic eradication of invasive and plantation of native species in the sanctuary are needed for better management.
\end{abstract}

\section{INTRODUCTION}

The Gangetic Plains in India form an important biogeographic zone characterized by fine alluvium and clay rich swamps, fertile soil and high water retention capacity. Around 400 species of angiosperms and 90120 species of aquatic plants have been recorded from this biogeographic zone. The landscape has the highest human density in India of 800-1200 people per $\mathrm{km}^{2}$, which has thus resulted in high human impact on natural ecosystems. The Gangetic Plains are scattered with several natural fresh water wetlands. Construction of irrigation canals, tanks, and dams with the primary purpose of irrigation and water supply for domestic purposes has also increased the number of wetlands across the region. In the course of time, these artificial wetlands attracted wildlife and therefore their conservation value has increased. It is estimated that freshwater wetlands alone support $20 \%$ of the known range of biodiversity in India (Deepa and Ramachandra 1999). The Okhla Bird Sanctuary (OBS) is one such wetland with a population of 14,000-20,000 water birds of 302 bird species recorded from the Sanctuary and its surrounding areas (Urfi 2003) and provides an example of conservation management of wetlands amidst a densely populated urban area.

Wetlands are often considered as 'hot spots' of biodiversity within a region or a landscape and support unique communities which involve a diversity of plants and animals (Gopal and Sah 1993) and are vastly studied for their functions as a habitat for birds (Stewart 2001). The number of bird species and their diversity is positively correlated with the richness of vegetation in and around a waterbody (Dvork and Best 1982; Andrikovics et al. 2006). Stewart (2001) suggested that the selection of habitats by birds depends on factors such as depth and quality of water, the availability of food, presence of vegetation for shelter, presence of predators and inter-species competitors. Vegetation establishes a strong association between aquatic and terrestrial ecosystems in a wetland, and botanical explorations of wetland plants are necessary to gain a comprehensive knowledge about the functioning of the system.

The course of the river Yamuna in Delhi is facing anthropogenic pressure leading to deteriorating water quality and changing water regime (Rawat et al. 2003; Aleem and Malik 2005; CPCB 2006; Trisal et al. 2008) and has a limited floodplain area because of embankments (Tabasum et al. 2009). The OBS has the widest floodplains within this stretch and provides protection to the floodplain habitat because of its status as a Sanctuary. Vegetation studies are fragmentary for the area. A total of 69 species were identified by Tabasum et al. (2009) from this stretch (till Okhla Barrage) that includes 39 aquatic and semi-aquatic and 30 terrestrial species. Another study (WII 2002) in OBS recorded 115 plants species belonging to 43 families. Urfi $(2003,2006)$ described the vegetation of the Sanctuary and surroundings in terms of bird habitat. Currently, a modern and more complete account is lacking despite the above such studies, and in particular a comprehensive list of plants of special relevance for management purposes has remained lacking. During the present study, OBS was under the authority of National Chambal Project Division, Agra, UP and a management plan for the same was being used for OBS, too. A plant checklist of Okhla Bird Sanctuary was prepared along with notes on the population status of some dominant aquatic and terrestrial species to overcome these information gaps.

\section{Materials ANd Methods \\ Area of study}

The Okhla Bird Sanctuary (Figure 1) is a flood plain wetland situated within the National Capital Region of Delhi, India and is part of protected area network of the state of Uttar Pradesh. Construction of the Okhla Barrage 


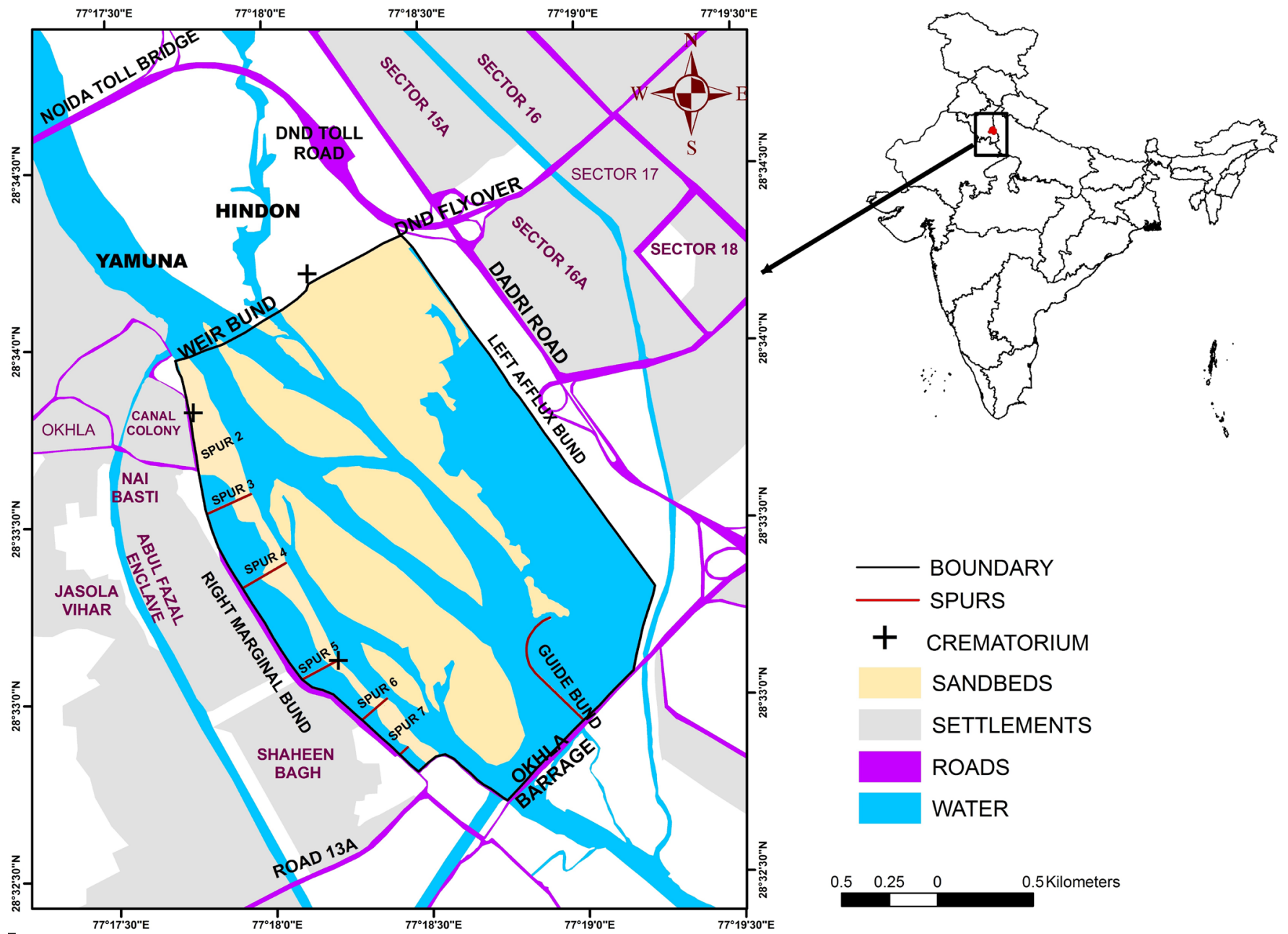

FIGURE 1. Map showing location of the Okhla Bird Sanctuary, National Capital Region, India.

across the river Yamuna has resulted in a small portion of the river to become a static water system. This wetland provides heterogeneous habitat for several migratory waterfowls, of which some species use the wetland as a stopover during their migration. The wetland was declared a Wildlife Sanctuary for Birds on 8 May, 1990 by the Uttar Pradesh government under the Wildlife (Protection) Act, 1972. It has an area of 400 ha with open water covering around 273 ha, reed and sand beds covering 97 ha and roads and bunds comprising the remaining 30 ha area. The area of the Sanctuary is a vast alluvial plain with a gentle southeastern slope. Well-metaled roads surround the Sanctuary and its boundaries are well defined by the mesh-wiring structures and bunds. Sediment of the reservoir is mainly composed of silt load carried by the river Yamuna. Sediment is black in color with slightly alkaline nature. The Sanctuary has mainly three seasons; summer (April-June, temperature range $40^{\circ} \mathrm{C}$ to $29^{\circ} \mathrm{C}$ ), winter (November-March, temperature range $21^{\circ} \mathrm{C}$ to $5^{\circ} \mathrm{C}$ ) and monsoon (July-September). In the metropolitan area of National Capital Region of India, this Sanctuary is valuable for recreation and conservation education.

\section{Methods}

Extensive floristic survey was performed to cover different micro-habitats in the Sanctuary, which included open water and shallow water areas, islands (sand-beds) within the river channel, floodplains and dykes between winter 2009 and spring 2010. Identification of plants in the field was made with the help of 'The Flora of Delhi' (Maheshwari 1963) and 'Trees of Delhi: A field guide' (Krishen 2006). Unidentified plants were brought to the Wildlife Institute of India (WII), Dehradun for further identification. Collected specimens were cross-checked and identified for correct identification at the Herbarium of WII. APG III (APG III 2009) classification was followed to classify the angiosperm species. To study weeds; all plant species exotic or native (but possessing prolific growth which may hamper the integrity of the ecosystem) were taken into account.

Percent cover for the submerged and floating plant species was estimated visually in $50 \times 50 \mathrm{~m}^{2}$ grids and averaged for the total area to see the extent of weeds. The sand-bed (islands and floodplains) vegetation was divided into upper stratum (mainly reeds) and lower stratum (various herbs). To study the frequency and density of terrestrial vegetation (tree species and amphibious species), $10 \times 10 \mathrm{~m}^{2}$ quadrates were laid at regular intervals of $100 \mathrm{~m}$ across the land surrounding the water body and islands.

\section{RESULT AND DisCUSSION}

A total of 192 species belonging to 46 families were recorded from the study area with Fabaceae (27 species), Poaceae (19 species), Asteraceae (17 species), Cyperaceae (15 species), Amaranthaceae (13 species), Malvaceae ( 9 
species), Convolvulaceae (8), Euphorbiaceae (7 species), Mimosaceae (7 species), Apocynaceae (7), Polygonaceae (6 species) and Moraceae ( 6 species) being the richest families, covering over $70 \%$ of the total species. Out of 192 species, 33 were trees, 10 were shrubs, 102 were herbs,
19 were grasses, 15 were sedges and 11 were climbers. Among pteridophytes, two species were recorded from the area. One species of a parasitic plant, Cuscuta reflexa was also recorded. Detailed vegetation characteristics of OBS are provided in Table 1.

TABLE 1. List of plant species from the Okhla Bird Sanctuary, National Capital Region of Delhi, Upper Gangetic Plains, India. (H=Herb; C=Climber; S=Shrub; T=Tree; Sd=Sedge; G=Grass; Aq: Aquatic; Tr: Terrestrial; Sm: Semi-aquatic; P: considered weed in present study; N: Considered weed only in previous study (WII 2002); P*: considered weed in both study).

\begin{tabular}{|c|c|c|c|c|}
\hline FAMILY/ SPECIES & HABIT & HABITAT & STATUS & VOUCHER NUMBER \\
\hline \multicolumn{5}{|l|}{ Araceae } \\
\hline Lemna perpusilla Torr. & $\mathrm{H}$ & $\mathrm{Aq}$ & & 11912 \\
\hline Spirodela polyrrhiza (L.) Schleid. & $\mathrm{H}$ & $\mathrm{Aq}$ & & 11919 \\
\hline \multicolumn{5}{|l|}{ Arecaceae } \\
\hline Phoenix sylvestris (L.) Roxb. & $\mathrm{T}$ & $\operatorname{Tr}$ & & 11890 \\
\hline \multicolumn{5}{|l|}{ Commelinaceae } \\
\hline Commelina forskalaei Vahl & $\mathrm{H}$ & $\operatorname{Tr}$ & & 11903 \\
\hline Commelina undulata R.Br. & $\mathrm{H}$ & $\mathrm{Tr} / \mathrm{Sm}$ & & 11876 \\
\hline \multicolumn{5}{|l|}{ Pontederiaceae } \\
\hline Eichhornia crassipes Solms & $\mathrm{H}$ & $\mathrm{Aq}$ & $\mathrm{P}^{*}$ & 11907 \\
\hline \multicolumn{5}{|l|}{ Typhaceae } \\
\hline Typha angustifolia $\mathrm{L}$. & $\mathrm{H}$ & $\operatorname{Tr} / \mathrm{Sm}$ & $\mathrm{P}^{*}$ & 11928 \\
\hline \multicolumn{5}{|l|}{ Cyperaceae } \\
\hline Carex alopecuroides D.Don ex Tilloch and Taylor & $\mathrm{Sd}$ & $\mathrm{Sm}$ & & 11902 \\
\hline Cyperus bulbosus Vahl & Sd & $\operatorname{Tr} / \mathrm{Sm}$ & & 11904 \\
\hline Cyperus compressus L. & $\mathrm{Sd}$ & $\operatorname{Tr}$ & & 11923 \\
\hline Cyperus iria $\mathrm{L}$. & $\mathrm{Sd}$ & $\operatorname{Tr} / \mathrm{Sm}$ & & 11922 \\
\hline Cyperus kyllingia Endl. & $\mathrm{Sd}$ & $\mathrm{Sm}$ & & 11905 \\
\hline Cyperus nutans Vahl & $\mathrm{Sd}$ & $\operatorname{Tr}$ & $\mathrm{N}$ & 11906 \\
\hline Cyperus triceps Endl. & $\mathrm{Sd}$ & $\mathrm{Sm}$ & & 11929 \\
\hline Eleocharis palustris (L.) Roem. and Schult. & Sd & $\mathrm{Sm}$ & & 11924 \\
\hline Fimbristylis cymosa subsp. cymosa & $\mathrm{Sd}$ & $\operatorname{Tr}$ & & 11937 \\
\hline Fimbristylis dichotoma (L.) Vahl & Sd & $\operatorname{Tr} / \mathrm{Sm}$ & & 11935 \\
\hline Fimbristylis ferruginea (L.) Vahl & $\mathrm{Sd}$ & $\operatorname{Tr} / \mathrm{Sm}$ & & 11909 \\
\hline Fimbristylis quinquangularis (Vahl) Kunth & $\mathrm{Sd}$ & $\operatorname{Tr}$ & & 11910 \\
\hline Scirpus littoralis Schrad. & $\mathrm{Sd}$ & $\mathrm{Aq}$ & $\mathrm{N}$ & 11932 \\
\hline \multicolumn{5}{|l|}{ Poaceae } \\
\hline Arundo donax L. & G & $\operatorname{Tr} / \mathrm{Sm}$ & & 11969 \\
\hline Avena sterilis L. & G & $\operatorname{Tr}$ & & 11971 \\
\hline Brachiaria distachya (L.) Stapf & G & $\operatorname{Tr}$ & & 11934 \\
\hline Brachiaria ramosa (L.) Stapf & G & $\mathrm{Tr} / \mathrm{Sm}$ & & 11960 \\
\hline Cenchrus ciliaris L. & G & $\operatorname{Tr}$ & & 11982 \\
\hline Cynodon dactylon (L.) Pers. & G & $\operatorname{Tr}$ & & 11961 \\
\hline Dactyloctenium aegyptium (L.) Willd. & G & $\operatorname{Tr}$ & & 11879 \\
\hline Dichanthium annulatum (Forssk.) Stapf & G & $\operatorname{Tr}$ & & 11880 \\
\hline Eragrostis pilosa (L.) P.Beauv. & G & $\operatorname{Tr}$ & & 11881 \\
\hline Imperata cylindrica (L.) Raeusch. & G & $\operatorname{Tr}$ & & 11962 \\
\hline Ischaemum indicum (Houtt.) Merrill & G & $\operatorname{Tr}$ & & 11891 \\
\hline Oplismenus burmannii (Retz.) P.Beauv. & G & $\operatorname{Tr}$ & & 11985 \\
\hline Paspalum distichum $\mathrm{L}$. & G & $\operatorname{Tr} / \mathrm{Sm}$ & & 11862 \\
\hline Pennisetum glaucum (L.) R.Br. & G & $\operatorname{Tr}$ & & 11979 \\
\hline Phragmites karka (Retz.) Trin. ex Steud. & G & $\operatorname{Tr} / \mathrm{Sm}$ & & 11931 \\
\hline Polypogon fugax Nees ex Steud. & G & $\operatorname{Tr}$ & & 11916 \\
\hline Saccharum bengalense Retz. & G & $\operatorname{Tr}$ & & 11838 \\
\hline Saccharum spontaneum L. & G & $\operatorname{Tr}$ & & 11839 \\
\hline Vetiveria zizanioides (L.) Nash & G & $\operatorname{Tr}$ & & 11958 \\
\hline \multicolumn{5}{|l|}{ Papaveraceae } \\
\hline Argemone mexicana L. & $\mathrm{H}$ & $\operatorname{Tr}$ & $\mathrm{P}^{*}$ & 11828 \\
\hline
\end{tabular}


TABle 1. Continued.

\begin{tabular}{|c|c|c|c|c|}
\hline FAMILY/ SPECIES & HABIT & HABITAT & STATUS & VOUCHER NUMBER \\
\hline Argemone ochroleuca Sweet & $\mathrm{H}$ & $\operatorname{Tr}$ & & 11956 \\
\hline \multicolumn{5}{|l|}{ Menispermaceae } \\
\hline Tinospora sinensis (Lour.) Merr. & $\mathrm{C}$ & $\operatorname{Tr}$ & & 11973 \\
\hline \multicolumn{5}{|l|}{ Ranunculaceae } \\
\hline Ranunculus sceleratus L. & $\mathrm{H}$ & $\operatorname{Tr} / \mathrm{Sm}$ & & 11977 \\
\hline \multicolumn{5}{|l|}{ Nelumbonaceae } \\
\hline Nelumbo nucifera Gaertn. & $\mathrm{H}$ & $\mathrm{Aq}$ & $\mathrm{N}$ & 11913 \\
\hline \multicolumn{5}{|l|}{ Proteaceae } \\
\hline Grevillea robusta A.Cunn. ex R.Br. & $\mathrm{T}$ & $\operatorname{Tr}$ & & 11811 \\
\hline \multicolumn{5}{|l|}{ Oxalidaceae } \\
\hline Oxalis corniculata $\mathrm{L}$. & $\mathrm{H}$ & $\operatorname{Tr}$ & & 11948 \\
\hline \multicolumn{5}{|l|}{ Euphorbiaceae } \\
\hline Acalypha indica L. & $\mathrm{H}$ & $\operatorname{Tr}$ & & 11843 \\
\hline Croton bonplandianus Baill. & $\mathrm{H}$ & $\operatorname{Tr}$ & & 11878 \\
\hline Euphorbia heterophylla L. & $\mathrm{H}$ & $\operatorname{Tr}$ & & 11974 \\
\hline Euphorbia granulata Forssk. & $\mathrm{H}$ & $\operatorname{Tr}$ & & 11978 \\
\hline Euphorbia hirta L. & $\mathrm{H}$ & $\operatorname{Tr}$ & & 11882 \\
\hline Kirganelia reticulata (Poir.) Baill. & $\mathrm{S}$ & $\operatorname{Tr}$ & & 11940 \\
\hline Ricinus communis L. & $\mathrm{H}$ & $\operatorname{Tr}$ & & 11855 \\
\hline \multicolumn{5}{|l|}{ Cucurbitaceae } \\
\hline Mukia maderaspatana (L.) M.Roem. & $\mathrm{C}$ & $\operatorname{Tr}$ & & 11853 \\
\hline Trichosanthes cucumerina $\mathrm{L}$. & $\mathrm{C}$ & $\operatorname{Tr}$ & & 11837 \\
\hline \multicolumn{5}{|l|}{ Fabaceae } \\
\hline Acacia nilotica (L.) Delile & $\mathrm{H}$ & $\operatorname{Tr}$ & & 11803 \\
\hline Albizia lebbeck (L.) Benth. & $\mathrm{H}$ & $\operatorname{Tr}$ & & 11871 \\
\hline Alhagi pseudalhagi (M. Bieb.) Desv. ex B. Keller and Shap. & $\mathrm{T}$ & $\operatorname{Tr}$ & & 11808 \\
\hline Alysicarpus vaginalis (L.) DC. & $\mathrm{H}$ & $\operatorname{Tr}$ & & 11976 \\
\hline Bauhinia purpurea L. & $\mathrm{T}$ & $\operatorname{Tr}$ & & 11847 \\
\hline Cassia tora $\mathrm{L}$. & $\mathrm{H}$ & $\operatorname{Tr}$ & & 11957 \\
\hline Cassia occidentalis L. & $\mathrm{H}$ & $\operatorname{Tr}$ & & 11991 \\
\hline Dalbergia sissoo DC. & $\mathrm{H}$ & $\operatorname{Tr}$ & & 11972 \\
\hline Delonix regia (Hook.) Raf. & $\mathrm{H}$ & $\operatorname{Tr}$ & & 11986 \\
\hline Desmodium triflorum (L.) DC. & $\mathrm{T}$ & $\operatorname{Tr}$ & & 11814 \\
\hline Enterolobium barinense Cardenas and Rodriguez & $\mathrm{T}$ & $\operatorname{Tr}$ & & 11966 \\
\hline Erythrina variegata $\mathrm{L}$. & $\mathrm{C}$ & $\operatorname{Tr}$ & & 11892 \\
\hline Indigofera hochstetteri Baker & $\mathrm{H}$ & $\operatorname{Tr}$ & & 11857 \\
\hline Indigofera linnaei Ali & $\mathrm{H}$ & $\operatorname{Tr}$ & & 11953 \\
\hline Leucaena leucocephala (Lam.) de Wit & $\mathrm{H}$ & $\operatorname{Tr}$ & & 11896 \\
\hline Melilotus alba Medik. & $\mathrm{T}$ & $\operatorname{Tr}$ & & 11984 \\
\hline Melilotus indica (L.) All. & $\mathrm{H}$ & $\operatorname{Tr}$ & & 11830 \\
\hline Parkinsonia aculeata L. & $\mathrm{H}$ & $\operatorname{Tr}$ & $\mathrm{N}$ & 11990 \\
\hline Pithecellobium dulce (Roxb.) Benth. & $\mathrm{T}$ & $\operatorname{Tr}$ & & 11988 \\
\hline Pongamia pinnata (L.) Pierre & $\mathrm{T}$ & $\operatorname{Tr}$ & & 11856 \\
\hline Prosopis cineraria (L.) Druce & $\mathrm{T}$ & $\operatorname{Tr}$ & & 11834 \\
\hline Prosopis juliflora (Sw.) DC. & $\mathrm{T}$ & $\operatorname{Tr}$ & & 11849 \\
\hline Rhynchosia minima (L.) DC. & $\mathrm{T}$ & $\operatorname{Tr}$ & & 11850 \\
\hline Tamarindus indica L. & $\mathrm{T}$ & $\operatorname{Tr}$ & $\mathrm{P}$ & 11946 \\
\hline Tephrosia pumila (Lam.) Pers. & $\mathrm{T}$ & $\operatorname{Tr}$ & & 11854 \\
\hline Tephrosia purpurea (L.) Pers. & $\mathrm{T}$ & $\operatorname{Tr}$ & & 11975 \\
\hline Tephrosia villosa (L.) Pers. & $\mathrm{T}$ & $\operatorname{Tr}$ & $\mathrm{P}$ & 11816 \\
\hline \multicolumn{5}{|l|}{ Rhamnaceae } \\
\hline Zizyphus mauritiana Lam. & $\mathrm{T}$ & $\operatorname{Tr}$ & & 11859 \\
\hline Zizyphus nummularia Wt. and Arn. & $\mathrm{S}$ & $\operatorname{Tr}$ & & 11822 \\
\hline \multicolumn{5}{|l|}{ Ulmaceae } \\
\hline Holoptelea integrifolia Planch. & $\mathrm{T}$ & $\operatorname{Tr}$ & & 11885 \\
\hline \multicolumn{5}{|l|}{ Cannabinaceae } \\
\hline Cannabis sativa $\mathrm{L}$. & $\mathrm{H}$ & $\operatorname{Tr}$ & $\mathrm{P}$ & 11868 \\
\hline \multicolumn{5}{|l|}{ Moraceae } \\
\hline Ficus benghalensis L. & $\mathrm{T}$ & $\operatorname{Tr}$ & & 11815 \\
\hline
\end{tabular}


TABle 1. Continued.

\begin{tabular}{|c|c|c|c|c|}
\hline FAMILY/ SPECIES & HABIT & HABITAT & STATUS & VOUCHER NUMBER \\
\hline Ficus benjamina $\mathrm{L}$. & $\mathrm{T}$ & $\operatorname{Tr}$ & & 11865 \\
\hline Ficus palmata Forssk. & S & $\operatorname{Tr}$ & & 11810 \\
\hline Ficus racemosa $\mathrm{L}$. & $\mathrm{T}$ & $\operatorname{Tr}$ & & 11851 \\
\hline Ficus religiosa L. & $\mathrm{T}$ & $\operatorname{Tr}$ & & 11840 \\
\hline Morus alba L. & $\mathrm{T}$ & $\operatorname{Tr}$ & & 11813 \\
\hline \multicolumn{5}{|l|}{ Myrtaceae } \\
\hline Syzygium cumini (L.) Skeels & $\mathrm{T}$ & $\operatorname{Tr}$ & & 11819 \\
\hline \multicolumn{5}{|l|}{ Salvadoraceae } \\
\hline Salvadora oleoides Decne. & S & $\operatorname{Tr}$ & & 11944 \\
\hline \multicolumn{5}{|l|}{ Cleomaceae } \\
\hline Cleome viscosa L. & $\mathrm{H}$ & $\operatorname{Tr}$ & & 11968 \\
\hline \multicolumn{5}{|l|}{ Brassicaceae } \\
\hline Coronopus didymus (L.) Sm. & $\mathrm{H}$ & $\operatorname{Tr}$ & & 11842 \\
\hline Rorippa nasturtium-aquaticum (L.)Hayek & $\mathrm{H}$ & $\mathrm{Aq} / \mathrm{Sm}$ & $\mathrm{N}$ & 11917 \\
\hline \multicolumn{5}{|l|}{ Malvaceae } \\
\hline Abutilon indicum (L.) Sweet & $\mathrm{S}$ & $\operatorname{Tr}$ & & 11801 \\
\hline Bombax ceiba L. & $\mathrm{T}$ & $\operatorname{Tr}$ & & 11806 \\
\hline Hibiscus micranthus L.f. & $\mathrm{H}$ & $\operatorname{Tr}$ & & 11877 \\
\hline Malva parviflora $\mathrm{L}$. & $\mathrm{H}$ & $\operatorname{Tr}$ & & 11895 \\
\hline Malvastrum coromandelianum (L.) Garcke & $\mathrm{H}$ & $\operatorname{Tr}$ & & 11992 \\
\hline Sida acuta Burm.f. & $\mathrm{H}$ & $\operatorname{Tr}$ & & 11894 \\
\hline Sida cordifolia L. & $\mathrm{H}$ & $\operatorname{Tr}$ & & 11817 \\
\hline Sida rhombifolia L. & $\mathrm{H}$ & $\operatorname{Tr}$ & & 11818 \\
\hline Urena lobata L. & $\mathrm{H}$ & $\operatorname{Tr}$ & $\mathrm{N}$ & 11858 \\
\hline \multicolumn{5}{|l|}{ Simaroubaceae } \\
\hline Ailanthus excelsa Roxb. & $\mathrm{T}$ & $\operatorname{Tr}$ & & 11870 \\
\hline \multicolumn{5}{|l|}{ Meliaceae } \\
\hline Azadirachta indica A.Juss. & $\mathrm{T}$ & $\operatorname{Tr}$ & & 11872 \\
\hline Melia azedarach L. & $\mathrm{T}$ & $\operatorname{Tr}$ & & 11863 \\
\hline \multicolumn{5}{|l|}{ Tamaricaceae } \\
\hline Tamarix dioica Roxb. & $\mathrm{S}$ & $\operatorname{Tr}$ & & 11820 \\
\hline \multicolumn{5}{|l|}{ Polygonaceae } \\
\hline Polygonum barbatum L. & $\mathrm{H}$ & $\mathrm{Tr} / \mathrm{Sm}$ & $\mathrm{N}$ & 11915 \\
\hline Polygonum glabrum Willd. & $\mathrm{H}$ & $\operatorname{Tr} / \mathrm{Sm}$ & & 11936 \\
\hline Polygonum hydropiper L. & $\mathrm{H}$ & $\operatorname{Tr} / \mathrm{Sm}$ & & 11926 \\
\hline Polygonum lapathifolia $\mathrm{L}$. & $\mathrm{H}$ & $\mathrm{Aq} / \mathrm{Sm}$ & & 11845 \\
\hline Polygonum plebeium R.Br. & $\mathrm{H}$ & $\operatorname{Tr}$ & & 11970 \\
\hline Rumex dentatus L. & $\mathrm{H}$ & $\operatorname{Tr}$ & & 11861 \\
\hline \multicolumn{5}{|l|}{ Amaranthaceae } \\
\hline Achyranthes aspera $\mathrm{L}$. & $\mathrm{H}$ & $\operatorname{Tr}$ & & 11848 \\
\hline Alternanthera paronychioides A.St.-Hil. & $\mathrm{H}$ & $\operatorname{Tr}$ & & 11827 \\
\hline Alternanthera philoxeroides (Mart.) Griseb. & $\mathrm{H}$ & $\operatorname{Tr} / \mathrm{Aq}$ & $\mathrm{P}$ & 11900 \\
\hline Alternanthera pungens Kunth & $\mathrm{H}$ & $\mathrm{Tr} / \mathrm{Sm}$ & $\mathrm{P}^{*}$ & 11804 \\
\hline Alternanthera sessilis (L.) R.Br. ex DC. & $\mathrm{H}$ & $\operatorname{Tr}$ & & 11805 \\
\hline Amaranthus tricolor $\mathrm{L}$. & $\mathrm{H}$ & $\operatorname{Tr}$ & & 11869 \\
\hline Amaranthus viridis $\mathrm{L}$. & $\mathrm{H}$ & $\operatorname{Tr}$ & & 11826 \\
\hline Chenopodium album $\mathrm{L}$. & $\mathrm{H}$ & $\operatorname{Tr}$ & & 11852 \\
\hline Chenopodium ambrosioides L. & $\mathrm{H}$ & $\operatorname{Tr}$ & & 11941 \\
\hline Chenopodium murale L. & $\mathrm{H}$ & $\mathrm{Tr} / \mathrm{Sm}$ & & 11920 \\
\hline Gomphrena celosioides Mart. & $\mathrm{H}$ & $\operatorname{Tr}$ & & 11987 \\
\hline Pupalia lappacea (L.) Juss. & $\mathrm{H}$ & $\operatorname{Tr}$ & & 11875 \\
\hline Suaeda maritima (L.) Dumort. & $\mathrm{H}$ & $\operatorname{Tr}$ & & 11833 \\
\hline \multicolumn{5}{|l|}{ Nyctaginaceae } \\
\hline Boerhavia diffusa $\mathrm{L}$. & $\mathrm{H}$ & $\operatorname{Tr}$ & & 11873 \\
\hline \multicolumn{5}{|l|}{ Basellaceae } \\
\hline Basella rubra L. & $\mathrm{C}$ & $\operatorname{Tr}$ & & 11829 \\
\hline \multicolumn{5}{|l|}{ Portulacaceae } \\
\hline Portulaca oleracea L. & $\mathrm{H}$ & $\operatorname{Tr}$ & & 11949 \\
\hline
\end{tabular}


TABle 1. Continued.

\begin{tabular}{|c|c|c|c|c|}
\hline FAMILY/ SPECIES & HABIT & HABITAT & STATUS & VOUCHER NUMBER \\
\hline \multicolumn{5}{|l|}{ Primulaceae } \\
\hline Anagallis arvensis L. & $\mathrm{H}$ & $\operatorname{Tr}$ & & 11938 \\
\hline \multicolumn{5}{|l|}{ Rubiaceae } \\
\hline Oldenlandia corymbosa L. & $\mathrm{H}$ & $\operatorname{Tr}$ & & 11947 \\
\hline \multicolumn{5}{|l|}{ Apocynaceae } \\
\hline Alstonia scholaris (L.) R. Br. & $\mathrm{T}$ & $\operatorname{Tr}$ & & 11823 \\
\hline Calotropis gigantea (L.) Dryand. & $\mathrm{T}$ & $\operatorname{Tr}$ & & 11874 \\
\hline Cascabela thevetia (L.) Lippold & $\mathrm{S}$ & $\operatorname{Tr}$ & & 11836 \\
\hline Dregea volubilis (L.f.) Benth. ex Hook.f. & $\mathrm{H}$ & $\operatorname{Tr}$ & & 11824 \\
\hline Oxystelma secamone K.Schum. & $\mathrm{C}$ & $\operatorname{Tr}$ & & 11959 \\
\hline Pergularia daemia (Forssk.) Chiov. & $\mathrm{C}$ & $\operatorname{Tr}$ & & 11930 \\
\hline Tabernaemontana divaricata (L.) R.Br. ex Roem. and Schult. & $\mathrm{C}$ & $\operatorname{Tr}$ & & 11967 \\
\hline \multicolumn{5}{|l|}{ Boraginaceae } \\
\hline Cordia dichotoma G.Forst. & $\mathrm{T}$ & $\operatorname{Tr}$ & & 11807 \\
\hline Ehretia laevis Roxb. & $\mathrm{T}$ & $\operatorname{Tr}$ & & 11809 \\
\hline \multicolumn{5}{|l|}{ Scrophulariaceae } \\
\hline Kickxia ramosissima Janchen & $\mathrm{H}$ & $\operatorname{Tr}$ & & 11886 \\
\hline Mazus pumilus (Burm. f.) Steenis & $\mathrm{H}$ & $\operatorname{Tr}$ & & 11888 \\
\hline \multicolumn{5}{|l|}{ Acanthaceae } \\
\hline Blepharis maderaspatensis (L.) B.Heyne & $\mathrm{H}$ & $\operatorname{Tr}$ & & 11939 \\
\hline Dicliptera paniculata (Forssk.) I.Darbysh. & $\mathrm{H}$ & $\operatorname{Tr}$ & & 11866 \\
\hline Peristrophe paniculata (Forssk.) Brumm. & $\mathrm{H}$ & $\operatorname{Tr}$ & & 11945 \\
\hline Rungia pectinata (L.) Nees & $\mathrm{H}$ & $\operatorname{Tr}$ & & 11846 \\
\hline \multicolumn{5}{|l|}{ Bignoniaceae } \\
\hline Kigelia africana (Lam.) Benth. & $\mathrm{T}$ & $\operatorname{Tr}$ & & 11812 \\
\hline Tecomella undulata (Sm.) Seem. & $\mathrm{S}$ & $\operatorname{Tr}$ & & 11860 \\
\hline \multicolumn{5}{|l|}{ Verbenaceae } \\
\hline Lantana camara L. & $\mathrm{S}$ & $\operatorname{Tr}$ & $\mathrm{P}^{*}$ & 11893 \\
\hline Phyla nodiflora (L.) Greene & $\mathrm{H}$ & $\operatorname{Tr} / \mathrm{Sm}$ & & 11914 \\
\hline \multicolumn{5}{|l|}{ Convolvulaceae } \\
\hline Cuscuta reflexa Roxb. & $\mathrm{H}$ & $\mathrm{P}$ & & 11832 \\
\hline Ipomoea aquatica Forssk. & $\mathrm{H}$ & $\mathrm{Aq}$ & & 11911 \\
\hline Ipomoea arachnosperma Welw. & $\mathrm{C}$ & $\operatorname{Tr}$ & & 11864 \\
\hline Ipomoea cairica (L.) Sweet & $\mathrm{C}$ & $\operatorname{Tr}$ & & 11884 \\
\hline Ipomoea fistulosa Mart. ex Choisy & $\mathrm{S}$ & $\operatorname{Tr} / \mathrm{Sm}$ & $\mathrm{P}^{*}$ & 11989 \\
\hline Ipomoea indica (Burm.) Merr. & $\mathrm{H}$ & $\mathrm{Tr} / \mathrm{Aq}$ & & 11963 \\
\hline Ipomoea sindica Stapf. & $\mathrm{C}$ & $\operatorname{Tr}$ & & 11955 \\
\hline Merremia aegyptia (L.) Urb. & $\mathrm{H}$ & $\operatorname{Tr}$ & & 11889 \\
\hline \multicolumn{5}{|l|}{ Solanaceae } \\
\hline Datura metel L. & $\mathrm{H}$ & $\operatorname{Tr}$ & & 11844 \\
\hline Nicotiana plumbaginifolia Viv. & $\mathrm{H}$ & $\operatorname{Tr}$ & & 11983 \\
\hline Solanum nigrum L. & $\mathrm{H}$ & $\operatorname{Tr}$ & & 11954 \\
\hline Solanum surattense Burm. f. & $\mathrm{H}$ & $\operatorname{Tr}$ & & 11965 \\
\hline Withania somnifera (L.) Dunal & $\mathrm{H}$ & $\operatorname{Tr}$ & & 11952 \\
\hline \multicolumn{5}{|l|}{ Asteraceae } \\
\hline Ageratum conyzoides (L.) L. & $\mathrm{H}$ & $\operatorname{Tr}$ & & 11802 \\
\hline Cirsium arvense (L.) Scop. & $\mathrm{H}$ & $\operatorname{Tr}$ & & 11831 \\
\hline Conyza canadensis (L.) Cronquist & $\mathrm{H}$ & $\operatorname{Tr}$ & & 11867 \\
\hline Cotula hemisphaerica Wall. ex Benth. and Hook.f. & $\mathrm{H}$ & $\operatorname{Tr}$ & & 11835 \\
\hline Eclipta prostrata (L.) L. & $\mathrm{H}$ & $\operatorname{Tr}$ & & 11897 \\
\hline Enydra fluctuans DC. & $\mathrm{H}$ & $\operatorname{Tr} / \mathrm{Sm}$ & & 11841 \\
\hline Gnaphalium pensylvanicum Willd. & $\mathrm{H}$ & $\mathrm{Aq} / \mathrm{Sm}$ & & 11908 \\
\hline Laggera aurita (DC.) & $\mathrm{H}$ & $\operatorname{Tr}$ & & 11883 \\
\hline Launaea nudicaulis (L.) Hook.f. & $\mathrm{H}$ & $\operatorname{Tr}$ & & 11981 \\
\hline Parthenium hysterophorus L. & $\mathrm{H}$ & $\operatorname{Tr}$ & & 11887 \\
\hline Pulicaria crispa Sch.Bip. & $\mathrm{H}$ & $\operatorname{Tr}$ & $\mathrm{P}^{*}$ & 11964 \\
\hline Soliva anthemifolia (Juss.) R.Br. ex Less. & $\mathrm{H}$ & $\operatorname{Tr}$ & & 11898 \\
\hline Sonchus arvensis $\mathrm{L}$. & $\mathrm{H}$ & $\operatorname{Tr}$ & & 11951 \\
\hline Tridax procumbens (L.) L. & $\mathrm{H}$ & $\operatorname{Tr}$ & & 11943 \\
\hline
\end{tabular}


TABLE 1. Continued.

\begin{tabular}{|c|c|c|c|c|}
\hline FAMILY/ SPECIES & HABIT & HABITAT & STATUS & VOUCHER NUMBER \\
\hline Vernonia cinerea (L.) Less. & $\mathrm{H}$ & $\mathrm{Tr}$ & & 11821 \\
\hline Xanthium strumarium L. & $\mathrm{H}$ & $\operatorname{Tr}$ & & 11899 \\
\hline Youngia japonica (L.) DC. & $\mathrm{H}$ & $\operatorname{Tr}$ & & 11942 \\
\hline \multicolumn{5}{|l|}{ Apiaceae } \\
\hline Centella asiatica (L.) Urb. & $\mathrm{H}$ & $\mathrm{Tr} / \mathrm{Sm}$ & & 11825 \\
\hline Hydrocotyle sibthorpioides Lam. & $\mathrm{H}$ & $\mathrm{Sm}$ & & 11925 \\
\hline \multicolumn{5}{|l|}{ Salviniaceae } \\
\hline Azolla pinnata R. Br. & Fern & $\mathrm{Aq}$ & $\mathrm{P}$ & 11918 \\
\hline Salvinia auriculata Aubl. & Fern & $\mathrm{Aq}$ & & 11901 \\
\hline
\end{tabular}

The previous study done in 2002 (WII 2002) had recorded 115 plant species belonging to 43 families from the Sanctuary. The major difference in both the lists is in number of herbs (addition of 49 species), tree species (addition of 18 species) and sedge (addition of 11 species). These changes can be attributed to variation in sampling seasons (changes in seasonal herbs) and difference in area covered in both the studies. The fluctuation in water levels of wetland results in difference in submergence of areas during various seasons; moreover, the sand-beds in the middle of the river channel are often submerged in water. This might have influenced the coverage of areas in the Sanctuary during 2002. Recent plantation by New Okhla Industrial Development Authority has also resulted in addition to the Sanctuary's flora.

\section{Wetland plant communities}

As the area is a storage water reservoir, water levels fluctuate with season and demand of water. This results in a varying water depth inside the Sanctuary, thus resulting in a number of aquatic vegetation communities in the area. Based on water depth, the Sanctuary had the following plant zones:

Areas with continuous standing water: These areas were largely dominated by two communities, viz., EichhorniaSalvinia-Spirodela, a free-floating plant community and Nelumbo-Eichhornia, a floating-leaved community. The former community is widely distributed in the Sanctuary and is dominated by Eichhornia crassipes, which forms large monotypic mats over water. At some places, E. crassipes was present with Alternanthera philoxeroides forming large continuous mats. The later community was restricted to only one location in the Sanctuary, some 30 ha in extent. It was dominated by a single species, Nelumbo nucifera, though with presence of E. crassipes. In the past, inhabitants of nearby human settlements used this area to harvest lotus fruits and flowers.

Waterlogged areas or areas with shallow water: These had primarily two communities; Alternanthera-PaspalumIpomoea in relatively shallow water areas and PhragmitesTypha-Saccharum-Arundo in waterlogged and draw down areas. Monotypic or intermixed patches of sedges e.g. Cyperus spp., Eleocharis palustris, Schoenoplectiella roylei were also present. The water body inside the Sanctuary had five islands (sand-beds), which were partially waterlogged and partially dry supporting diverse emergent vegetation communities. These islands had varying water depths, none of which were completely dry during the study. They had plant communities with an upper stratum comprising reeds, sedges and cattail and a lower stratum of herbs, short grasses and other aquatic macrophytes. Reed or tall grass species included Phragmites karka, Saccharum spp. and Arundo donax. The most abundant species in upper stratum was Typha angustifolia forming a continuous closed stand in permanently waterlogged or submerged habitats. This species is not tolerant to dry soils and is very successful in waterlogged conditions. It has colonized many shallower areas with increasing water depth and had the greatest coverage in the Sanctuary (Figure 2). Typha elephantina was present in several small patches. T. elephantina that flourishes in dry, upland and even saline zones by virtue of deeply-established rhizomes, was usually found nearer to banks. Both the species are common in the floodplains of the river Yamuna. Saccharum spp. formed large clumps in comparatively drier areas. Saccharum spontaneum is commonly found on alluvial soils and can tolerate infrequent flooding, but never grows in water or frequent flooding regimes (Dabadhagao and Shankarnayana 1973). Accordingly, it remained confined to relatively drier, peripheral areas of the wetland at higher elevation. Saccharum spp. formed a community with Typha spp., particularly T. elephantina in one of the islands. Phragmites karka and Arundo donax formed either monotypic patches or patches having both the species. Both the species are well adapted to flooding and were abundant in the areas with continuous heavy floods followed by dry phase (Innes 1977; Mackenzie 2004). Sedges present included Eleocharis palustris, Schoenoplectiella roylei, Carex alopecuroides and Cyperus spp. These formed small monotypic or inter-mixed stands. Sedge like E. palustris formed distinct patches in areas with permanent water.

The lower stratum was dominated by Paspalum distichum and Alternanthera spp. (Figure 3). Paspalum distichum grew well both near water and in lower marshy areas. The growth of $P$. distichum is reported to vary depending upon water depth and grazing pressures. In a study in Keoladeo National Park, Middleton (1990) observed maximum growth (shoot density and length) of $P$. distichum in water-saturated soils, though the biomass was greater under flooded conditions. In the Sanctuary, it occurred over a wide water depth. Alternanthera spp. were the most frequent among all genera, occurring in $75 \%$ plots (Table 2). Alternanthera sessilis and Alternanthera philoxeroides were the most frequently occurring species. The percent occurrence of the above-mentioned species in 
the Sanctuary is given in Table 2. Ipomoea spp. were also present in shallow water and dry areas; Ipomoea fistulosa being the most frequent in this genera.

\section{Tree species}

A total of 33 species of trees had been recorded during current study. Prosopis juliflora, Leucaena leucocephala, Dalbergia sissoo, Morus alba and Zizyphus mauritiana were most frequent species among the trees observed in the Sanctuary. The frequency and density (individuals ha${ }^{1}$ ) of major tree species from OBS are given in Table 3. It was observed that Leucaena leucocephala had the highest density (192.8 individuals $\mathrm{ha}^{-1}$ ), followed by Prosopis juliflora (142.8 individuals ha ${ }^{-1}$ ), Dalbergia sissoo (85.7 individuals $\mathrm{ha}^{-1}$ ) and Morus alba (57.1 individuals ha $\left.{ }^{1}\right)$. L. lucocephela has covered many parts of high ground (mainly embankments such as afflux bund and guide bund) in the Sanctuary. There are many large trees of L. leucocephala and thick natural regeneration is seen under and near them. P. juliflora grows in some of the high areas on the boundary of the OBS. It is not regenerating independently and hence does not pose much problem currently. A number of trees (both indigenous and exotic) have been planted particularly inside the Sanctuary on the eastern side of the road running on the left afflux bund by the New Okhla Industrial Development Authority e.g. Alstonia scholaris, Bauhinia purpurea, Melia azedarach. However, most of these species are not added in the list as the work was in progress during the present study, and nothing can be said with surety regarding the survival of these owing to their early stage.

\section{Invasive plants/weed infestation}

Weed infestation has caused major problems for many ecosystems. These plants, often exotic species, grow aggressively and cause changes in the habitat (Janick 1979). Thirteen major weeds were identified in OBS during the study of which five species were amphibious (Typha angustifolia, Typha elephantina, Alternanthera sessilis, Alternanthera philoxeroides and Ipomoea fistulosa), two were aquatic (Eichhornia crassipes and Salvinia auriculata) and six were terrestrial (Parthenium hysterophorus, Lantana camara, Cannabis sativa, Argemone mexicana, Leucaena leucocephala and Prosopis juliflora). A study in 2002 (WII 2002) had recorded 22 weeds from the area. Out of these, 5 aquatic and 1 terrestrial species viz. Nymphaea nouchali, Trapa bispinosa, Marsilea quqdrifoliata, Utricularia inflexa var. stellaris, Bacopa monnieri and Eclipta prostrata, respectively, were not recorded during current study. There was an addition of 6 new weed species in our list, while 9 species considered weeds in the previous study were not included here, as these did not match the criteria of weeds followed by us (Table 1). At present, OBS is facing threats from the rapid proliferation of Typha angustifolia and Eichhornia crassipes. About 70\% of sample plots in the terrestrial and shallower habitats of OBS were dominated by the species of Typha, with approximately 65.88 ha of the Sanctuary covered by Typha dominated vegetation. Most of this area was earlier good shallow water habitats for dabbling ducks and waders; a

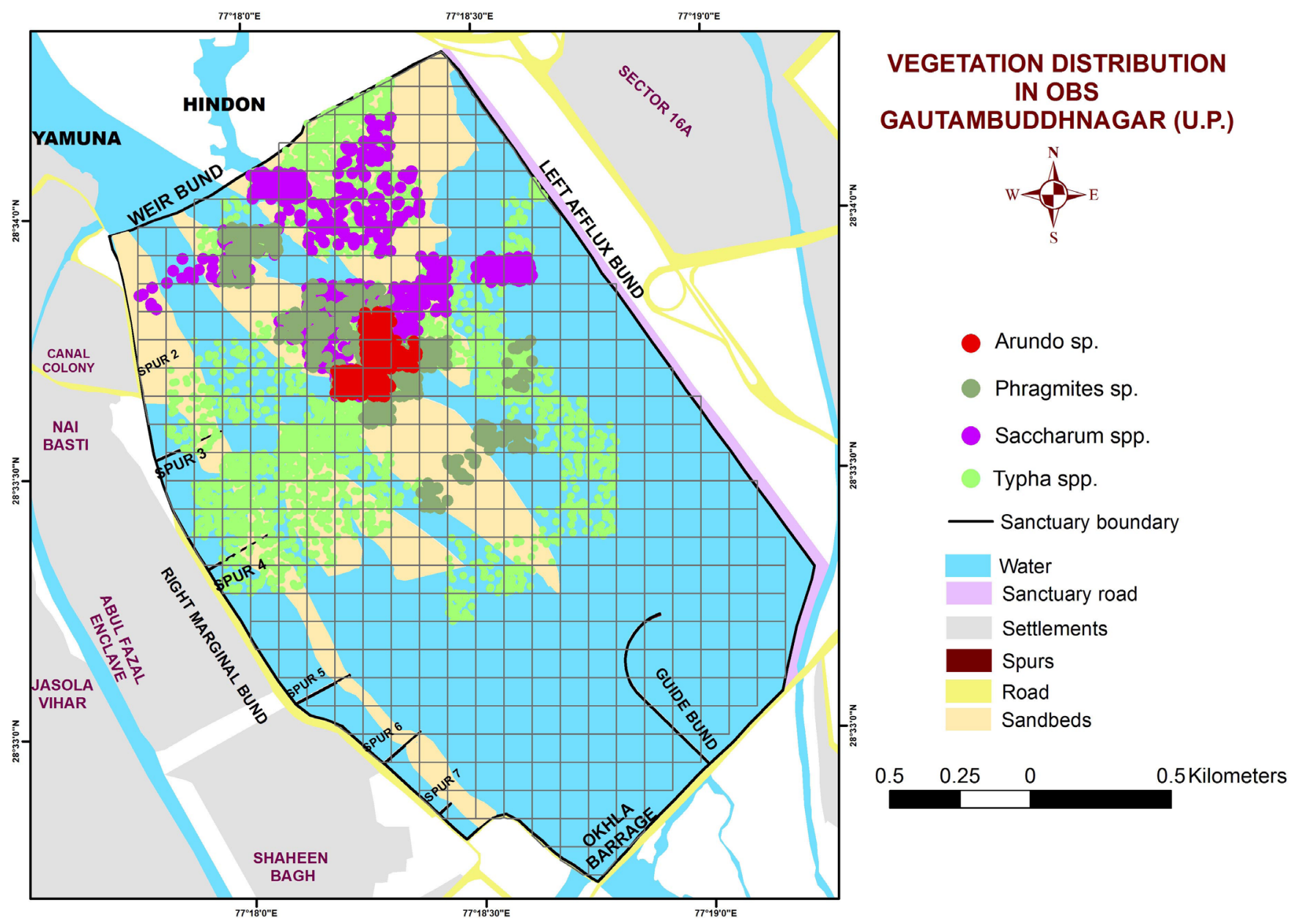

FIGURE 2. Map showing dominant species of upper stratum in draw-down areas and islands of the Okhla Bird Sanctuary, National Capital Region, India. 
habitat which has almost vanished from OBS (personal communication with regular birdwatchers). During the present study, the extent of weeds varied from $20 \%$ in December to $70 \%$ in May (Figure 4). It was observed that weeds (and particularly aquatic species) proliferated with the advent of the summer season. This may be a function of increased nutrient level in water during summer.

Expansion of aquatic weeds and their highly fluctuating biomass pose threats to habitats of waterbirds and native vegetation. Steps need to be taken to check the ingress of Typha into the areas which are still free from it. Certain areas from where Typha has to be removed on priority have been identified in the present study and are mentioned in the management plan (WII 2011). These include areas where Typha angustifolia has encroached in recent times and areas which were known as shallow habitat for birds

TABLE 2. Percent occurrence of major amphibious plant species of the Okhla Bird Sanctuary during current study.

\begin{tabular}{ll}
\hline PLANT SPECIES & \% PLOTS \\
\hline Alternanthera spp. & 75.0 \\
Typha angustifolia & 40.0 \\
Paspalum distichum & 33.0 \\
Typha elephantina & 30.0 \\
Saccharum bengalense & 28.3 \\
Phragmites karka & 26.2 \\
Arundo donax & 12.4 \\
Saccharum spontaneum & 12.2 \\
Ipomoea fistulosa & 6.5 \\
\hline
\end{tabular}

in the past. A suggested strategy to remove T. angustifolia from these areas is to cut it below the water level, which would lead to dying (rotting) of root stock and should ideally be done in the small post-monsoon time window, well before arrival of winter migratory birds.

Both Leucaena leucocephala and Prosopis juliflora are exotic species in India. Leucaena leucocephala is native to southern Mexico and northern Central America and has now

TABLE 3. Major tree species and their frequency (\%) and density (individuals ha-1) in Okhla Bird Sanctuary during current study.

\begin{tabular}{llll}
\hline FAMILY & SPECIES & FREQUENCY & DENSITY \\
\hline Mimosaceae & Prosopis juliflora & 78.6 & 142.9 \\
Mimosaceae & Leucaena leucocephala & 35.7 & 192.9 \\
Fabaceae & Dalbergia sissoo & 32.1 & 85.7 \\
Moraceae & Morus alba & 32.1 & 57.1 \\
Rhamnaceae & Zizyphus mauritiana & 25.0 & 32.1 \\
Meliaceae & Azadirachta indica & 17.9 & 17.9 \\
Caesalpiniaceae & Bauhinia purpurea & 17.9 & 21.4 \\
Bombacaceae & Bombax ceiba & 14.3 & 14.3 \\
Mimosaceae & Albizia lebbeck & 10.7 & 39.3 \\
Mimosaceae & Pithecellobium dulce & 10.7 & 21.4 \\
Fabaceae & Erythrina variegata & 7.1 & 7.1 \\
Moraceae & Ficus religiosa & 7.1 & 7.1 \\
Ulmaceae & Holoptelea integrifolia & 7.1 & 10.7 \\
Moraceae & Ficus benghalensis & 3.6 & 3.6 \\
Bignoniaceae & Kigelia pinnata & 3.6 & 3.6 \\
Fabaceae & Parkinsonia aculeata & 3.6 & 3.6 \\
Fabaceae & Pongamia glabra & 3.6 & 3.6 \\
\hline
\end{tabular}

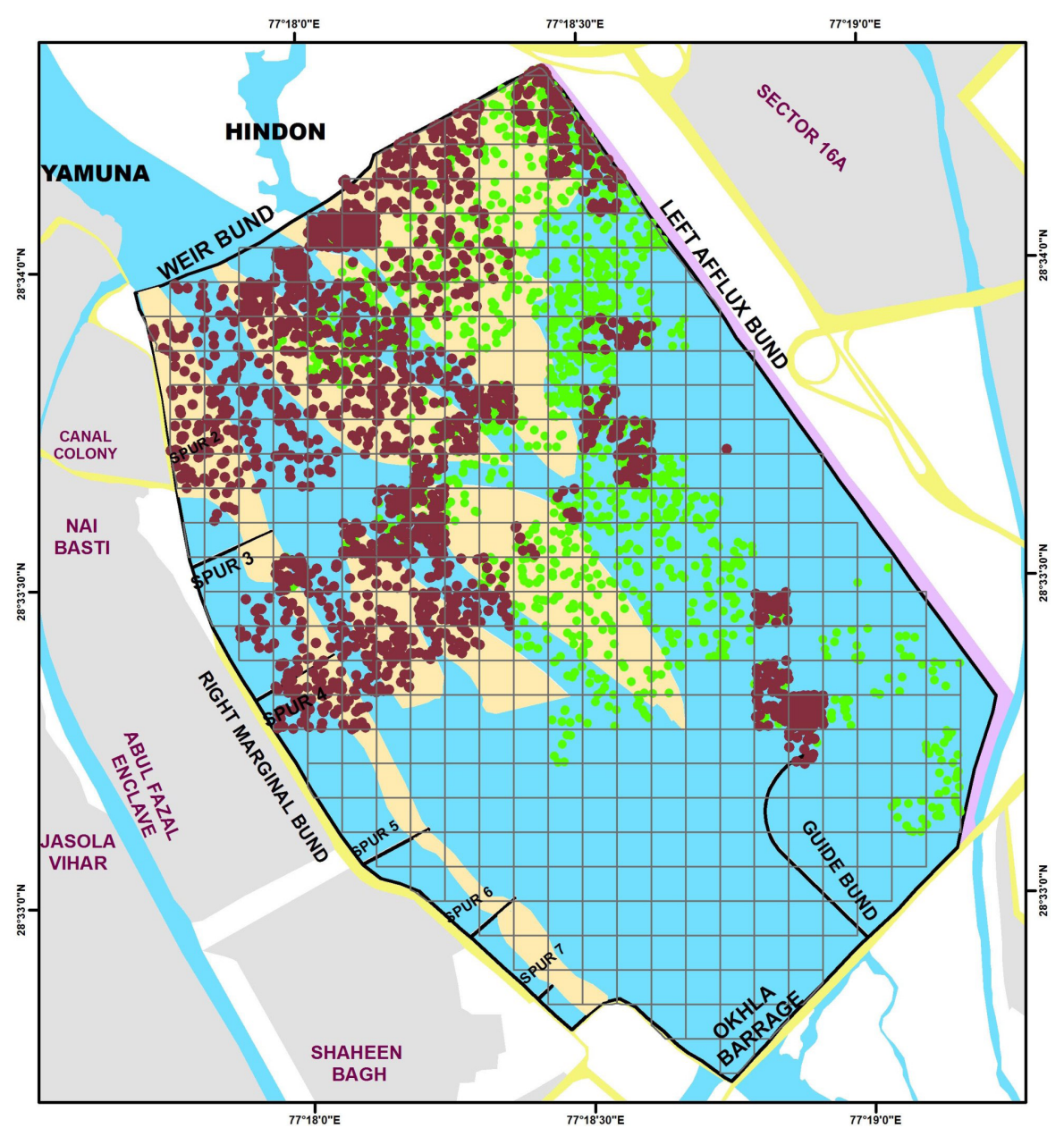

\section{VEGETATION DISTRIBUTION IN OBS \\ GAUTAMBUDDHNAGAR (U.P.)}<smiles>N=c1c(N)c(F)c1=S</smiles>

- Paspalum sp.

- Alternanthera spp.

Sanctuary boundary

Water

Settlements

Spurs

Road

Sandbeds
Sanctuary road

FIGURE 3. Map showing spread of Paspalum distichum and Alternanthera spp. in the Okhla Bird Sanctuary, National Capital Region, India. 
naturalized throughout the tropics. It is highly invasive in the arid climates, growing quickly and resulting in dense thickets which crowd out native vegetation. It is considered one of the 100 worst invasive species by the Invasive Species Specialist Group of the IUCN Species Survival Commission (Global Invasive Species Database 2010). Prosopis juliflora is native to Mexico, South America and the Caribbean Islands and has established as a weed in Asia, Australia and elsewhere. It is considered a noxious invader. These species have covered many parts of elevated ground (mainly embankments such as afflux bund, guide bund and spurs on the western bank) in the Sanctuary.

Importance of vegetation in the Sanctuary as bird habitat

A total of 302 species of birds have been reported from the area from several records since 1989 (Urfi 2003). Thus conservation of plant species and communities important for bird nesting and feeding in the area are of prime concern. The Sanctuary has extensive reed beds, shallow vegetated areas, and open deep water habitats. Tall grasses and vegetation like Phragmites karka, Saccharum spp., Typha spp., and sedges along the wetland and in the drawdown areas provide shelter and protection to bird species like Anser indicus, Tadorna ferruginea, Ardea purpurea, Pseudibis papillosa, Mycteria leucocephala etc. Presence of extensive reed cover and floating vegetation provides ideal habitat for bird species e.g. Gallinula chloropus, Porphyrio porphyrio, Ardea cinerea and Ardeola grayii. Tall grasses and other vegetation act as a buffer against disturbance from roads surrounding the Sanctuary and provide an ideal escape for waterfowl. The NelumboEichhornia community provides a good roosting habitat for waterfowl. However, large, unwanted monotypic stands of Eichhornia crassipes and continuous monotypic stands of Typha angustifolia have reduced the value of the wetland as good waterfowl habitat.

Grasses like Brachiaria spp., Avena sterilis, Cenchrus ciliaris, Dactyloctenium aegyptium etc. provide food for the birds of the genera Lonchura, Prinia, Francolinus etc., while sedges like Cyperus spp., Eleocharis sp. etc. also provide food to waterfowl and other birds. Trees like Ficus spp., Morus alba, Syzygium cumini, Zizyphus mauritiana, Ehretia laevis, Pithecellobium dulce etc., herbaceous climbers like Mukia maderaspatana etc. and shrubs like Zizyphus nummularia are also good sources of food for birds. However, the two most frequent and abundant tree species (L. leucocephala and P. juliflora) are exotic to the area and indigenous plant species like Ficus spp., Morus alba, Acacia nilotica, Zizyphus spp. etc. should be planted more in the area. The thick regeneration of $L$. leucocephala needs to be uprooted from most terrestrial areas to eliminate it gradually from OBS. The process of removal of $L$. leucocephala and $P$. juliflora should be done at a later stage once native trees are properly established. No plantations should however be raised on the riverine stretches of the Sanctuary.

Due to a high pressure on floodplain wetlands, the remaining stretches should be recognized as ecologically fragile and included in land-use planning. The role played by native vegetation of the region in maintaining ecological functioning should be integrated in management actions or river restoration plans. Regular monitoring of vegetation change, invasive species extension and its effect on native species should be done. OBS has a mosaic of habitats that is responsible for a rich avifauna. There is a need to monitor these habitats for long-term protection and conservation of various groups of flora and fauna in the Sanctuary. The present findings can be used as a baseline for future studies and a comparison with previous works suggests that steps should be taken to curtail the growth of weeds and plantation of native species should be encouraged.

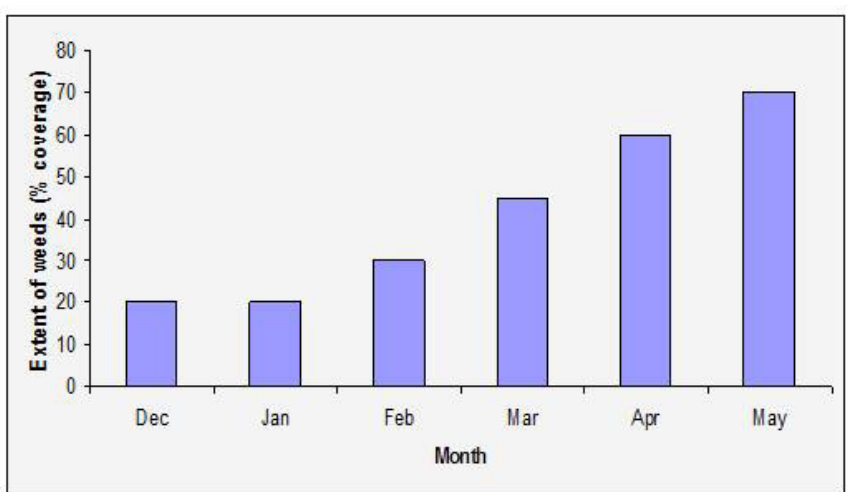

FIGURE 4. Extent of weeds (\% of plots covered) in the Sanctuary in different months between December 2009-May 2010, Okhla Bird Sanctuary, National Capital Region, India.

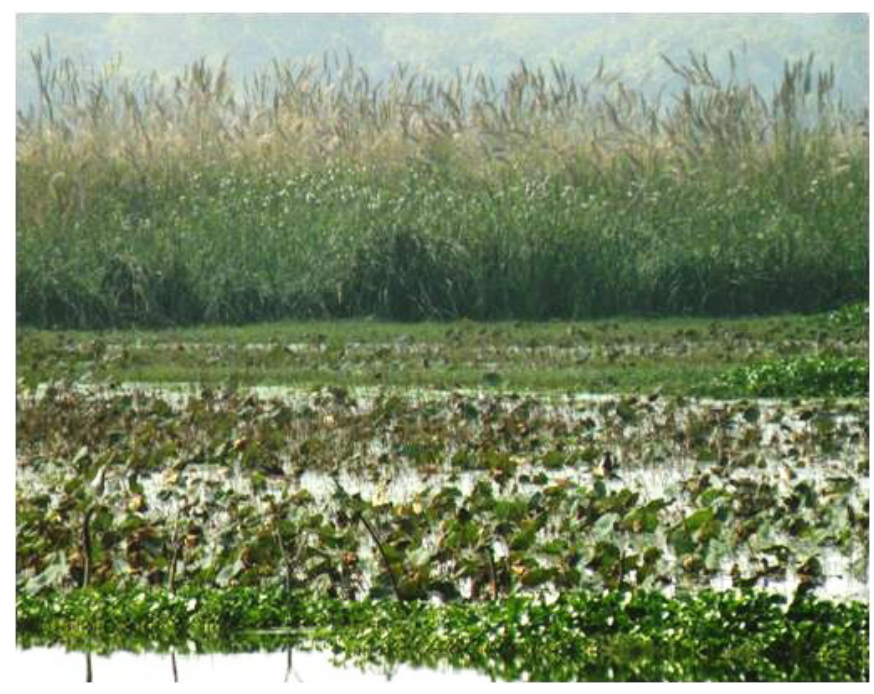

FIGURE 5. Nelumbo - Eichhornia, a floating-leaved community with reed beds in the background of Okhla Bird Sanctuary, National Capital Region, India.

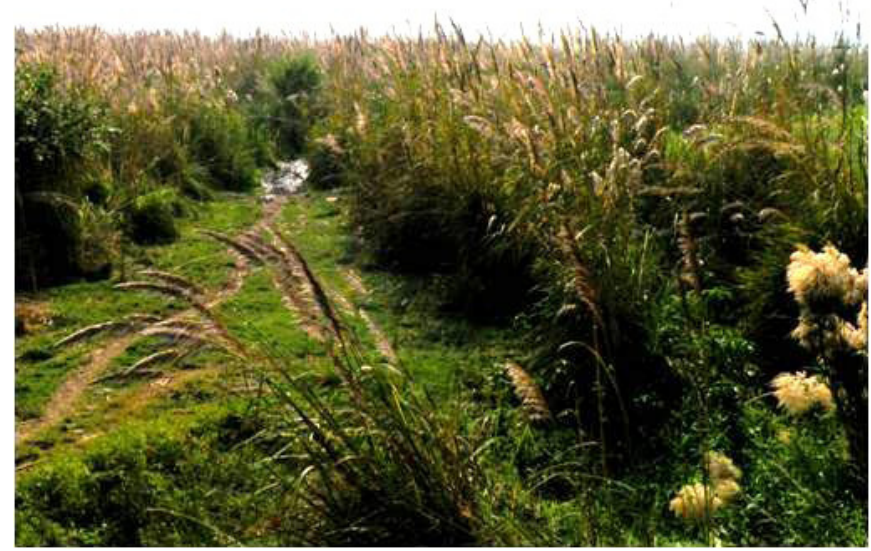

FIGURE 6. Reed beds of Phragmites - Arundo on one of the islands of Okhla Bird Sanctuary, National Capital Region, India. 


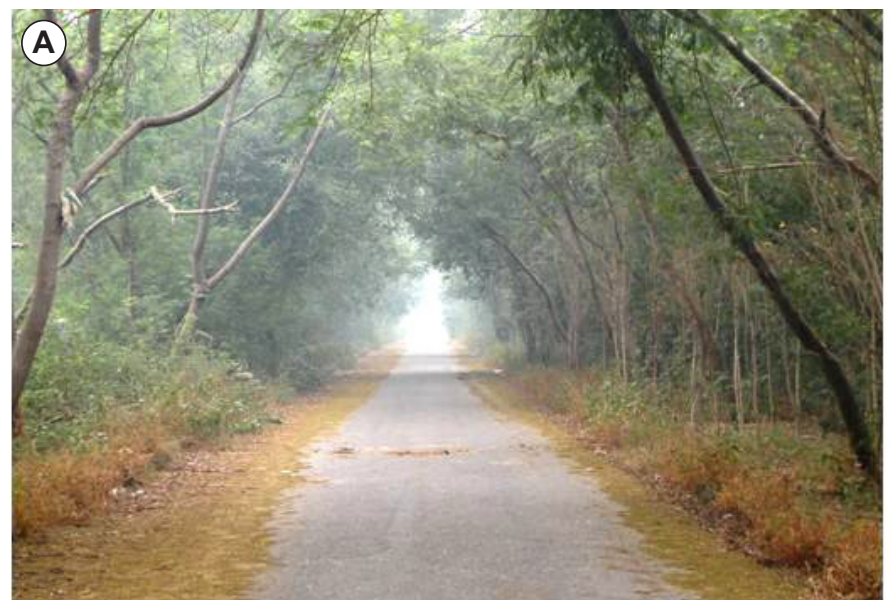

(B)

FIGURE 7. Weed spread in the Sanctuary (A) Leucaena leucocephala stand B) Large mats of Eichhornia crassipes in Okhla Bird Sanctuary, National Capital Region, India.
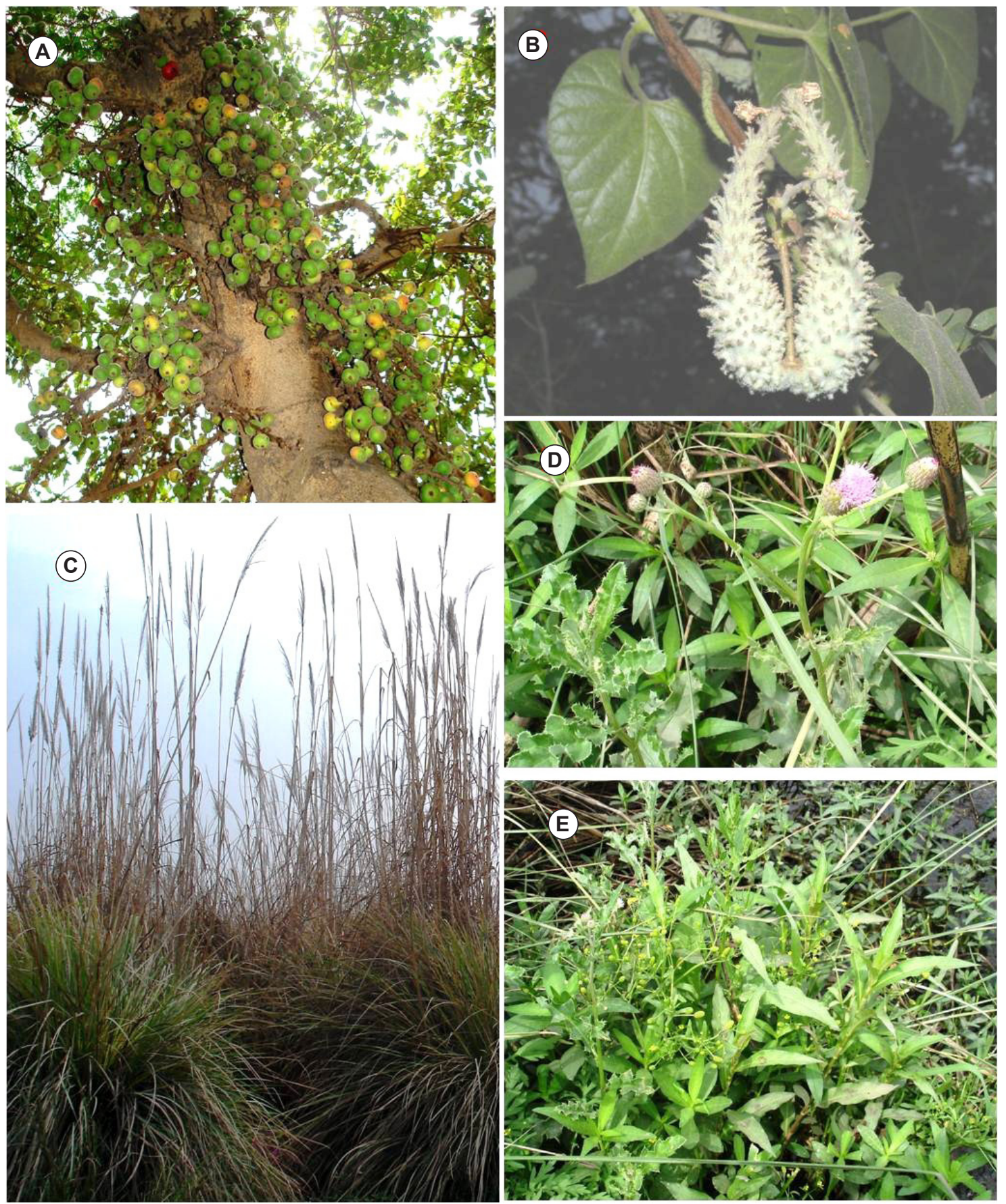

FIGURE 8. (A) Ficus recemosa (B) Pergularia daemia (C) Saccharum bengalense (D) Cirsium arvense (E) Ranunculus sceleratus in Okhla Bird Sanctuary, National Capital Region, India. 
ACKNowledgments: We are grateful to the Director and Dean, WII, Dehra Dun, for providing necessary facilities to carry out this work and Forest department of Uttar Pradesh for permission and their support in the field. We also like to thank anonymous reviewers and Dr. Gajendra Singh and Amit Kumar at WII for critical reviews and valuable suggestions.

\section{Literature Cited}

Aleem, A. and A. Malik. 2005. Genotoxicity of the Yamuna River water at Okhla (Delhi), India. Ecotoxicology and Environment Safety 61: 404412.

Andrikovics, S., L. Forró, G. Gere, G. Lakatos and L. Sasvári. 2006. Water bird guilds and their feeding connections in the Bodrogzug, Hungary. Hydrobiologia 567(1): 31-42.

APG III. 2009. An update of the Angiosperm Phylogeny Group classification for the orders and families of flowering plants: APG III. Botanical Journal of the Linnean Society 161:105-121.

Central Pollution Control Board (CPCB). 2006. Water quality status of Yamuna River (1999 - 2005). Assessment and Development of River Basin Series: ADSORBS/41/2006-07. Accessible at http://www. cpcb.nic.in/newitems/11.pdf. Captured on 25 January 2012.

Dabadhagao, P.M. and K.A. Shankarnarayana. 1973. The Grass Cover of India. New Delhi: Indian Council of Agricultural Research.

Deepa, R.S. and T.V. Ramachandra. 1999. Impact of Urbanization in the interconnectivity of wetlands. Proceedings of the National Symposium on Remote Sensing Applications for Natural Resources: Retrospective and perspective: 343-351.

Dvork, J. and E.P.H. Best. 1982. Macro-invertebrate communities associated with the macrophytes of Lake Vechten: structural and functional relationships. Hydrobiologia 95: 115-126.

Global Invasive Species Database. 2010. Leucaena leucocephala (tree). Electronic database available at http://www.issg.org/database/ species/ecology. Captured on 19 January 2012.

Gopal, B. and M. Sah. 1993. Conservation and management of rivers in India: case study of the River Yamuna. Environmental Conservation 20: $243-254$.

Innes, R.R. 1977. A manual of Ghana grasses. Chatham: NRI. 265 p.

Janick, J. 1979. Horticultural Science (3rd ed.). San Francisco: W.H. Freeman. 308 p.

Krishen, P. 2006. Trees of Delhi: A field Guide. Delhi: Penguin Books. 360 p.

Mackenzie, A. 2004. Giant Reed. In C. Harrington and A. Hayes (ed.). The Weed Workers' Handbook. Accessible at http://www.cal-ipc.org/ip/ management/wwh/pdf/19646.pdf. Captured on 19 January 2012
Maheshwari, J.K. 1963. The Flora of Delhi. New Delhi: Council of scientific and Industrial Research. 447 p.

Middleton, B.A. 1990. Effect of water depth and clipping frequency on the growth and survival of four wetland plant species. Aquatic Botany 37: 189-196.

Rawat M, Moturi MCZ, Subramanian V. 2003. Inventory compilation and distribution of heavy metals in wastewater from small-scale industrial areas of Delhi, India. Journal of Environment Monitoring 5: 906-912.

Stewart, R.E.Jr. 2001. Technical Aspects of Wetlands: Wetlands as Bird Habitats. National Water Summery on Wetland Resources. United States Geological Survey Water Supply Paper 2425. Accessible at http://water.usgs.gov/nwsum/WSP2425/ birdhabitat.html. Captured on 2 May 2009.

Tabasum, T., P. Bhat, R. Kumar, T. Fatma and C.L. Trisal. 2009. Vegetation of the river Yamuna floodplain in the Delhi stretch, with reference to hydrological characteristics. Ecohydrology 2: 156-163.

Trisal, C., T. Tabassum and R. Kumar. 2008. Water quality of the river Yamuna in the Delhi stretch: key determinants and management issues. Clean 36: 306-314.

Urfi, A. J. 2003. The birds of Okhla Bird Sanctuary, Delhi, India. Forktail 19: 39-50.

Urfi A J. 2006. Biodiversity conservation in an urban landscape: a case study of some important bird areas on the river Yamuna in Delhi (India); p. 303-317 In J.A. McNeely, T.M. McCarthy, A. Smith, L. OlsvigWhittaker and E.D. Wikramanayake (ed.). Conservation Biology in Asia. Kathmandu: The Society for Conservation Biology Asia Section and Resources Himalaya.

WII. 2002. Ecological, social and hydrological factors affecting the management of wetland systems in Uttar Pradesh with special reference to Vijay Sagar and associated water bodies in Mahoba district, Okhla and associated water bodies in Ghaziabad district, Bakhira Bird Sanctuary, and Nawabganj Bird Sanctuary. Dehra Dun: Wildlife Institute of India. $147 \mathrm{p}$.

WII. 2011. Management plan for Okhla Bird Sanctuary (2011 to 2021). Dehra Dun: Wildlife Institute of India. 217 p.

RECEIVED: April 2012

ACCEPTED: February 2013

Published ONLINE: April 2013

EDITORIAL RESPONSIBILITY: Paul Egan 